\title{
Avaliação da qualidade de vida com o instrumento SF-36 durante a pandemia do COVID-19: Um estudo piloto
}

\author{
Quality of life assessment with the SF-36 instrument during the COVID-19 pandemic: A pilot study \\ Evaluación de la calidad de vida con el instrumento SF-36 durante la pandemia de COVID-19: Un \\ estudio piloto
}

Recebido: 27/06/2021 | Revisado: 04/07/2021 | Aceito: 13/07/2021 | Publicado: 19/07/2021

Roberta de Oliveira e Silva
ORCID: https://orcid.org/0000-0002-9243-1478
Universidade Federal de Alfenas, Brasil
Centro de saúde especializado, Brasil
E-mail: robs1006oliveira @ gmail.com
Jéssica Naiara Pereira
ORCID: https://orcid.org/0000-0003-3309-1490
Centro de saúde especializado, Brasil
E-mail: naiarajessica814@ @mail.com
Eliana Gazana Pereira Milan
ORCID: https://orcid.org/0000-0003-3941-0835
Centro de saúde especializado, Brasil
E-mail: elianaenfermagemg@gmail.com

\section{Resumo}

O objetivo deste trabalho foi avaliar a qualidade de vida de voluntários saudáveis através do questionário SF-36 por contato telefônico durante a pandemia do COVID-19 no município de Machado/MG. O SF-36 é um questionário multidimensional formado por 36 itens, englobados em oito escalas ou componentes: capacidade funcional, aspectos físicos, dor, estado geral de saúde, vitalidade, aspectos sociais, aspectos emocionais, saúde mental e mais uma questão de avaliação comparativa entre as condições de saúde atual e há de um ano atrás. Avalia tanto os aspectos negativos da saúde (doença ou enfermidade), como os aspectos positivos (bem-estar). Foram entrevistados 114 voluntários entre novembro de 2020 e janeiro de 2021. Desses, 52 (45,6\%) eram do sexo feminino e $62(54,4 \%)$ eram do sexo masculino. A idade média da amostra estudada foi de 34,99 anos (DP 12,01). A análise estatística foi feita através do programa estatístico SPSS versão 2.0. Foi utilizado o teste t independente para a comparação de média e desvio padrão entre os gêneros e os domínios do questionário SF-36, e o coeficiente de correlação de Pearson classificando a correlação em perfeita $(r=1)$, forte $(r>0,75)$, moderada $(r>0,5)$, fraca $(r<0,5)$ e inexistente $(r=0)$. $O$ nível de significância para o teste foi de 5\%. Não houve diferença significativa entre os sexos feminino e masculino nos domínios, porém os aspectos emocionais destacam-se por apresentar média maior no sexo masculino e, houve diferença significativa entre alguns domínios quando correlacionados, porém com correlação de Pearson linear positiva fraca.

Palavras-chave: Qualidade de vida; Voluntários saudáveis; Inquéritos e questionários.

\begin{abstract}
The objective of this study was to evaluate the quality of life of healthy volunteers through the SF-36 questionnaire by telephone contact during the COVID-19 pandemic in the city of Machado/MG. The SF-36 is a multidimensional questionnaire composed of 36 items, encompassed in eight scales or components: functional capacity, physical aspects, pain, general health status, vitality, social aspects, emotional aspects, mental health, and one more question comparing current health conditions to those of one year ago. It assesses both the negative aspects of health (illness or disease) and the positive aspects (well-being). 114 volunteers were interviewed between November 2020 and January 2021. Of these, $52(45.6 \%)$ were female and $62(54.4 \%)$ were male. The mean age of the sample studied was 34.99 years (SD 12.01). Statistical analysis was done using the statistical program SPSS version 2.0. The independent t-test was used for the comparison of mean and standard deviation between genders and the domains of the SF-36 questionnaire, and Pearson's correlation coefficient classified the correlation as perfect $(r=1)$, strong ( $r>0.75)$, moderate $(r>0.5)$, weak $(r<0.5)$ and non-existent $(r=0)$. The significance level for the test was $5 \%$. There was no significant difference between female and male sexes in the domains, but the emotional aspects stand out for presenting a higher mean in males and there was a significant difference between some domains when correlated, but with a weak positive linear Pearson correlation.
\end{abstract}

Keywords: Quality of life; Healthy volunteers; Surveys and questionnaires. 


\begin{abstract}
Resumen
El objetivo de este estudio fue evaluar la calidad de vida de voluntarios sanos mediante el cuestionario SF-36 por contacto telefónico durante la pandemia de COVID-19 en la ciudad de Machado/MG. El SF-36 es un cuestionario multidimensional compuesto por 36 ítems, englobados en ocho escalas o componentes: capacidad funcional, aspectos físicos, dolor, estado de salud general, vitalidad, aspectos sociales, aspectos emocionales, salud mental y una pregunta más de evaluación comparativa entre las condiciones de salud actuales y hace un año. Evalúa tanto los aspectos negativos de la salud (enfermedad o dolencia) como los positivos (bienestar). Se entrevistó a 114 voluntarios entre noviembre de 2020 y enero de 2021. De ellos, $52(45,6 \%)$ eran mujeres y $62(54,4 \%)$ eran hombres. La edad media de la muestra estudiada era de 34,99 años (DE 12,01). El análisis estadístico se realizó con el programa estadístico SPSS versión 2.0. Se utilizó la prueba t independiente para la comparación de la media y la desviación estándar entre los géneros y los dominios del cuestionario SF-36, y el coeficiente de correlación de Pearson clasificó la correlación como perfecta $(r=1)$, fuerte $(r>0,75)$, moderada $(r>0,5)$, débil $(r<0,5)$ e inexistente $(r=0)$. El nivel de significación de la prueba fue del $5 \%$. No hubo diferencias significativas entre los sexos femenino y masculino en los hogares, por lo que los aspectos emocionales se destacan por presentarse en mayor medida en el sexo masculino y, hubo diferencias significativas entre algunos hogares cuando se correlacionan, por lo que con la correlación de Pearson fraco positiva lineal.
\end{abstract}

Palabras clave: Calidad de vida; Voluntarios sanos; Consultas y cuestionarios.

\title{
1. Introdução
}

O novo Coronavírus (2019-nCoV) é um vírus identificado como a causa de um surto de doença respiratória detectado pela primeira vez em Wuhan, China (Mcintosh, 2020). No entanto, sabe-se que o vírus tem alta transmissibilidade e provoca uma síndrome respiratória aguda que varia de casos leves, cerca de 80\%, a casos muito graves com insuficiência respiratória, entre $5 \%$ e $10 \%$ dos casos. Sua letalidade varia, principalmente, conforme a faixa etária e condições clínicas associadas (Ministério da saúde, 2020).

Em 30 de janeiro de 2020, a Organização Mundial da Saúde (OMS) declarou o surto do COVID-19 (Doença Coronavírus 2019) uma emergência de saúde pública e, em 11 de março, a epidemia foi atualizado para pandemia (World Health Organization, 2020).

Gradativamente o vírus foi despertando em nós o sentimento de medo e muitas outras preocupações. Meses depois do conhecimento público sobre a coexistência do novo coronavírus com a população brasileira, o cenário tornou-se vertiginoso e assolador (Moretti, et al., 2020).

Podemos definir é que o novo coronavírus nos trouxe inúmeros e significativos rompimentos. Sem muita cerimônia, a COVID-19 rompeu com a nossa conhecida rotina. Desde então, nossas agendas pré-programadas perderam o sentido. Nosso cotidiano, mentalmente organizado, sofreu alterações. E, neste novo cenário, incertezas e inseguranças foram acionadas ou intensificadas. Surge então, o medo e a desconfiança. Medo de ser contaminado, de adoecer e de morrer; medo de ter sua renda reduzida ou mesmo eliminada; medo de perder alguém estimado ou que este convalesça em sofrimento; medo de que o período de isolamento se estenda para além do qual o sujeito imagina que possa suportar. Seja qual a forma de medo causado pela COVID-19, este está sempre acompanhado de ameaças (Moretti, et al., 2020).

Com o avanço da tecnologia, as formas de percepção e interação com o mundo mudaram e a crise ocasionada pela pandemia do COVID-19 trouxe consigo uma revolução digital, já que o isolamento social se tornou um fator essencial no enfrentamento da disseminação da doença, com regras rígidas de bloqueio e distanciamento social muitos países adotaram essas práticas. Deste modo, embora esse aumento no uso de ferramentas digitais fosse previsto e estivesse crescendo nos últimos anos, o vírus catalisou o processo que pessoas e empresas insistiam em postergar (Oliveira, et al., 2020).

Inicialmente, o Covid-19 foi identificado apenas como um problema respiratório, mas com o aumento do número de casos ficou evidente que o vírus afeta uma variedade de sistemas, havendo relatos de complicações cardíacas, renais, hematológicas e neurológicas nos estados mais avançados, podendo inclusive levar à falência múltipla dos órgãos. Sabe-se 
também que pacientes que apresentam comorbidades prévias, são mais susceptíveis ao desenvolvimento de formas mais graves da doença, principalmente diabéticos e portadores de doenças cardiovasculares (Chatterjee, 2020; Vetta, et al., 2020).

A telessaúde é definida como o uso da tecnologia da informação e dos meios de telecomunicação para a realização de ações de saúde à distância e a telemedicina é uma subárea da telessaúde que visa o fornecimento de atenção médica a pacientes sem o contato físico. As estratégias de telemedicina são ideais para facilitar o gerenciamento de pacientes na ausência de consultas presenciais e a eficácia desse método tem sido amplamente avaliada (Poulter, et al., 2015; Casonatto \& Doederlein, 2009). A relevância de ferramentas de telessaúde tornou-se evidente durante a pandemia, sendo necessários novos estudos para validar seus métodos e assegurar a eficácia de tratamentos (Bashshur, et al., 2020; Rockwell \& Gilroy, 2020).

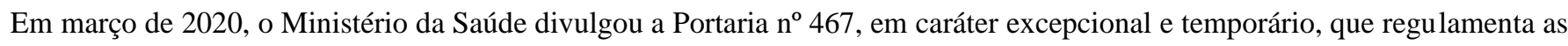
ações de telemedicina, permitindo o atendimento pré-clínico, de suporte assistencial, monitoramento e diagnóstico, por meio de tecnologia da informação e comunicação, no âmbito do Sistema Único de Saúde (SUS), bem como na saúde suplementar e privada (Ministério da saúde, 2020).

Para avaliar a qualidade de vida foi utilizado o questionário SF-36 (Short-Form Health Survey). Este instrumento de qualidade de vida multidimensional foi desenvolvido em 1992 por Ware e Sherbourne e validado no Brasil por Ciconelli et al. A avaliação dos resultados foi feita mediante a atribuição de escores para cada questão, os quais foram transformados numa escala de zero a 100, onde zero correspondeu a uma pior qualidade de vida e 100 a uma melhor qualidade de vida (Adorno \& Brasil-Neto, 2013). É um questionário de fácil entendimento que pode ser aplicado via contato telefônico.

Diante deste cenário, o objetivo deste trabalho foi avaliar a qualidade de vida de voluntários saudáveis através do instrumento SF-36 por contato telefônico durante a pandemia do COVID-19.

No contexto atual, o atendimento virtual permite o tratamento de pacientes infectados sem a necessidade de contato físico, possibilitando consultas e intervenções personalizadas via telefone, conexões de Internet ao vivo e sessões prégravadas (Hollander \& Carr, 2020). Embora seja necessária a realização de mais estudos quanto ao impacto econômico da utilização da telessaúde, pesquisas até o momento demonstram um custo-benefício favorável a essa forma de intervenção em comparação ao modelo tradicional de forma presencial (Tousignant, et al., 2015; Pastora-Bernal, et al., 2017; Nelson, et al., 2019; Cottrell, et al., 2019).

Devido à pandemia do COVID-19 declarada como emergência em saúde pública pela Organização Mundial da Saúde (OMS) em 11 de março de 2020 (World Health Organization, 2020), este trabalho foi realizado via contato telefônico resguardando os pesquisadores e voluntários do contato social e prevenindo possíveis contaminações.

\section{Metodologia}

Trata-se de um estudo transversal, quantitativo (Dalfovo, 2008) com a aplicação do questionário SF-36 em voluntários saudáveis durante a pandemia do COVID-19. Foram entrevistados 114 voluntários via contato telefônico no município de Machado/MG entre novembro de 2020 e janeiro de 2021. Os critérios de inclusão foram voluntários auto relatados saudáveis, com idade entre 18 e 70 anos, de ambos os sexos e que assinaram o termo de consentimento livre e esclarecido (TCLE) sendo informados quanto aos objetivos, à importância das atividades desenvolvidas e os possíveis resultados. Foram excluídos indivíduos com alguma patologia física, metabólica ou cognitiva, que não compreendiam a leitura e interpretação e que não possuíam clareza cognitiva. O estudo foi aprovado pelo Comitê de Ética e Pesquisa da União das Instituições de Serviço, Ensino e Pesquisa - UNISEPE, através do parecer nº 4.376.599, CAAE: 38634020.9.0000.5490.

O questionário SF-36 (Short-Form Health Survey) é um instrumento de qualidade de vida (QV) multidimensional que foi desenvolvido em 1992 por Ware e Sherbourne e validado no Brasil por Ciconelli et al, formado por 36 itens englobados em oito escalas ou componentes: capacidade funcional (10 itens), aspectos físicos (quatro itens), dor (dois itens), estado geral de 
saúde (cinco itens), vitalidade (quatro itens), aspectos sociais (dois itens), aspectos emocionais (três itens), saúde mental (cinco itens) e mais uma questão de avaliação comparativa entre as condições de saúde atual e há de um ano atrás. Avalia tanto os aspectos negativos da saúde (doença ou enfermidade), como os aspectos positivos (bem-estar).

Para avaliação de seus resultados, após sua aplicação, é dado um escore para cada questão que posteriormente são transformados numa escala de 0 a 100, onde zero corresponde a um pior estado de saúde e 100 a um melhor, sendo analisada cada dimensão em separado. Propositalmente, não existe um único valor que resuma toda a avaliação, traduzindo-se num estado geral de saúde melhor ou pior, justamente para que, numa média de valores, evite-se o erro de não se identificar os verdadeiros problemas relacionados à saúde do paciente ou mesmo de subestimá-los.

A análise estatística foi feita através do programa estatístico SPSS versão 2.0 (SPSS Inc., Chicago, IL, USA). A análise descritiva foi apresentada em média e desvio padrão (DP). O teste de normalidade para as variáveis estudadas indicou distribuição normal dos dados pelo teste de Kolmogorov-Smirnov, o que permitiu a utilização de teste paramétrico para os dados. Foi utilizado o teste $\mathrm{t}$ independente para a comparação de média e desvio padrão entre os gêneros e os domínios do questionário SF-36, e o coeficiente de correlação de Pearson classificando a correlação em perfeita $(r=1)$, forte $(r>0,75)$, moderada $(r>0,5)$, fraca $(r<0,5)$ e inexistente $(r=0)$. O nível de significância para o teste foi de $5 \%$.

\section{Resultados e Discussão}

As características demográficas estão apresentadas na Tabela 1. Dos 114 voluntários entrevistados, 52 (45,6\%) eram do sexo feminino e $62(54,4 \%)$ eram do sexo masculino. A idade média da amostra estudada foi de 34,99 anos (DP 12,01). Todos os voluntários foram capazes de responder o questionário de forma completa e não houve desistência na participação.

Tabela 1 - Características demográficas.

\begin{tabular}{cc}
\hline $\mathbf{N}$ & $\mathbf{1 1 4}$ \\
\hline Feminino (\%) & $52(45,6 \%)$ \\
Masculino (\%) & $62(54,4 \%)$ \\
Idade média (DP) & $34,99(12,01)$ \\
\hline
\end{tabular}

Estatística descritiva (SPSS 2.0). Fonte: Autores.

A Tabela 2 mostra os valores obtidos de média e desvio padrão para cada domínio do questionário SF-36. Cada domínio apresenta um escore final de zero a 100, no qual zero corresponde ao pior estado geral de saúde e 100, ao melhor estado de saúde. 
Tabela 2 - Valores obtidos para cada domínio do questionário SF-36.

\begin{tabular}{lcc}
\hline DOMíNIOS & MÉDIA & DESVIO PADRÃO \\
\hline Capacidade funcional & 78,68 & 17,49 \\
\hline Aspectos físicos & 71,93 & 37,50 \\
\hline Dor & 70,28 & 16,63 \\
\hline Estado geral da saúde & 55,35 & 9,65 \\
Vitalidade & 59,12 & 13,14 \\
\hline Aspectos sociais & 59,94 & 17,74 \\
\hline Aspectos emocionais & 82,16 & 62,42 \\
\hline Saúde mental & 55,72 & 12,36 \\
\hline
\end{tabular}

Estatística descritiva (SPSS 2.0). Fonte: Autores.

A tabela 3 apresenta a comparação de média e desvio padrão entre os gêneros e os domínios do questionário SF-36. Não houve diferença significativa entre os sexos feminino e masculino nos domínios, porém os aspectos emocionais destacamse por apresentar média maior no sexo masculino.

Tabela 3 - Comparação entre gênero nos domínios do questionário SF-36.

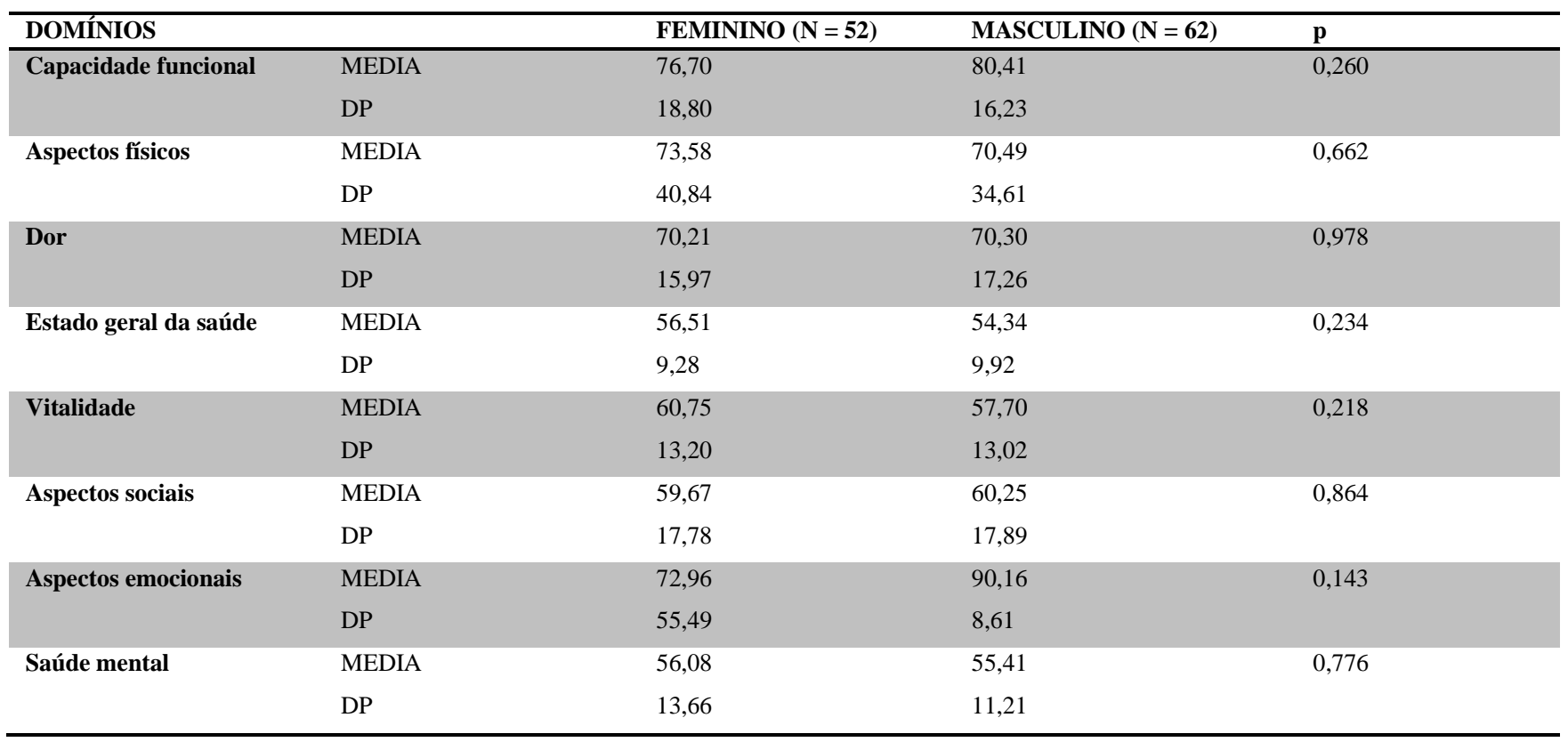

Teste $\mathrm{T}$ independente. $\mathrm{p}<0,05$. Fonte: Autores.

A Tabela 4 apresenta a correlação entre os domínios do questionário SF-36. O domínio capacidade funcional relacionado aos domínios aspectos físicos, dor, estado geral da saúde, aspectos sociais e saúde mental apresentam significância estatística, porém com correlação de Pearson linear positiva fraca , assim como o domínio aspectos físicos relacionado com os 
domínios capacidade funcional, vitalidade, aspectos emocionais e saúde mental; o domínio dor relacionado com os domínios capacidade funcional, vitalidade, aspectos sociais e saúde mental; o domínio estado geral da saúde relacionado com os domínios capacidade funcional, vitalidade, aspectos sociais e saúde mental; o domínio vitalidade relacionado com os domínios aspectos físicos, dor, estado geral da saúde, aspectos sociais e aspectos emocionais; o domínio aspectos sociais relacionado com os domínios capacidade funcional, dor, estado geral da saúde, vitalidade e saúde mental; o domínio aspectos emocionais relacionado com os domínios aspectos físicos e o domínio saúde mental relacionado com os domínios capacidade funcional, aspectos físicos, dor, estado geral da saúde, vitalidade e aspetos sociais.

Os domínios dor e aspectos emocionais assim como os domínios aspectos sociais e aspectos emocionais apresentam significância estatística, porém com correlação de Pearson fraca linear negativa ou inversa.

Tabela 4 - Correlação entre os domínios do questionário SF-36.

\begin{tabular}{|c|c|c|c|c|c|c|c|c|}
\hline & $\begin{array}{l}\text { Capacidade } \\
\text { funcional }\end{array}$ & $\begin{array}{c}\text { Aspectos } \\
\text { físicos }\end{array}$ & Dor & $\begin{array}{c}\text { Estado geral } \\
\text { da saúde }\end{array}$ & Vitalidade & $\begin{array}{c}\text { Aspectos } \\
\text { sociais }\end{array}$ & $\begin{array}{c}\text { Aspectos } \\
\text { emocionais }\end{array}$ & $\begin{array}{l}\text { Saúde } \\
\text { mental }\end{array}$ \\
\hline \multirow{2}{*}{$\begin{array}{l}\text { Capacidade } \\
\text { funcional }\end{array}$} & $\mathrm{r}=1$ & $\mathrm{r}=0,263$ & $\mathrm{r}=0,157$ & $\mathrm{r}=0,252$ & $\mathrm{r}=0,057$ & $r=0,224$ & $\mathrm{r}=0,113$ & $\mathrm{r}=0,456$ \\
\hline & & $\mathrm{p}=0,005$ & $\mathrm{p}=0,095$ & $\mathrm{p}=0,007$ & $\mathrm{p}=0,544$ & $\mathrm{p}=0,017$ & $\mathrm{p}=0,230$ & $\mathrm{p}=0,000$ \\
\hline \multirow[t]{2}{*}{ Aspectos físicos } & $r=0,263$ & $\mathrm{r}=1$ & $\mathrm{r}=-, 020$ & $\mathrm{r}=0,037$ & $\mathrm{r}=0,185$ & $\mathrm{r}=0,092$ & $\mathrm{r}=0,260$ & $\mathrm{r}=0,204$ \\
\hline & $\mathrm{p}=0,005$ & & $\mathrm{p}=0,835$ & $\mathrm{p}=0,694$ & $\mathrm{p}=0,048$ & $\mathrm{p}=0,330$ & $\mathrm{p}=0,005$ & $\mathrm{p}=0,029$ \\
\hline \multirow[t]{2}{*}{ Dor } & $\mathrm{r}=0,157$ & $r=-0,020$ & $\mathrm{r}=1$ & $\mathrm{r}=0,100$ & $\mathrm{r}=0,192$ & $\mathrm{r}=0,485$ & $r=-0,413$ & $\mathrm{r}=0,221$ \\
\hline & $\mathrm{p}=0,095$ & $\mathrm{p}=0,835$ & & $\mathrm{p}=0,289$ & $\mathrm{p}=0,041$ & $\mathrm{p}=0,000$ & $\mathrm{p}=0,000$ & $\mathrm{p}=0,018$ \\
\hline \multirow{2}{*}{$\begin{array}{l}\text { Estado geral da } \\
\text { saúde }\end{array}$} & $\mathrm{r}=0,252$ & $\mathrm{r}=0,037$ & $\mathrm{r}=0,100$ & $\mathrm{r}=1$ & $\mathrm{r}=0,232$ & $\mathrm{r}=0,215$ & $\mathrm{r}=0,111$ & $\mathrm{r}=0,188$ \\
\hline & $\mathrm{p}=0,007$ & $\mathrm{p}=0,694$ & $\mathrm{p}=0,289$ & & $\mathrm{p}=0,013$ & $\mathrm{p}=0,021$ & $\mathrm{p}=0,238$ & $\mathrm{p}=0,045$ \\
\hline \multirow[t]{2}{*}{ Vitalidade } & $\mathrm{r}=0,057$ & $\mathrm{r}=0,185$ & $\mathrm{r}=0,192$ & $\mathrm{r}=0,232$ & $\mathrm{r}=1$ & $\mathrm{r}=0,206$ & $\mathrm{r}=0,067$ & $\mathrm{r}=0,470$ \\
\hline & $\mathrm{p}=0,544$ & $\mathrm{p}=0,048$ & $\mathrm{p}=0,041$ & $\mathrm{p}=0,013$ & & $\mathrm{p}=0,028$ & $\mathrm{p}=0,478$ & $\mathrm{p}=0,000$ \\
\hline \multirow[t]{2}{*}{ Aspectos sociais } & $\mathrm{r}=0,224$ & $\mathrm{r}=0,092$ & $\mathrm{r}=0,485$ & $\mathrm{r}=0,215$ & $\mathrm{r}=0,206$ & $\mathrm{r}=1$ & $r=-0,337$ & $\mathrm{r}=0,232$ \\
\hline & $\mathrm{p}=0,017$ & $\mathrm{p}=0,330$ & $\mathrm{p}=0,000$ & $\mathrm{p}=0,021$ & $\mathrm{p}=0,028$ & & $\mathrm{p}=0,000$ & $\mathrm{p}=0,013$ \\
\hline \multirow{2}{*}{$\begin{array}{l}\text { Aspectos } \\
\text { emocionais }\end{array}$} & $\mathrm{r}=0,113$ & $\mathrm{r}=0,260$ & $r=-, 413$ & $\mathrm{r}=0,111$ & $\mathrm{r}=0,067$ & $r=-0,337$ & $\mathrm{r}=1$ & $\mathrm{r}=-0,057$ \\
\hline & $\mathrm{p}=0,230$ & $\mathrm{p}=0,005$ & $\mathrm{p}=0,000$ & $\mathrm{p}=0,238$ & $\mathrm{p}=0,478$ & $\mathrm{p}=0,000$ & & $\mathrm{p}=0,547$ \\
\hline \multirow[t]{2}{*}{ Saúde mental } & $\mathrm{r}=0,456$ & $\mathrm{r}=0,204$ & $\mathrm{r}=0,221$ & $\mathrm{r}=0,188$ & $\mathrm{r}=0,470$ & $\mathrm{r}=0,232$ & $r=-0,057$ & $\mathrm{r}=1$ \\
\hline & $\mathrm{p}=0,000$ & $\mathrm{p}=0,029$ & $\mathrm{p}=0,018$ & $\mathrm{p}=0,045$ & $\mathrm{p}=0,000$ & $\mathrm{p}=0,013$ & $\mathrm{p}=0,547$ & \\
\hline
\end{tabular}

Correlação de Pearson(r). $r=1$ perfeita, $r>0,75$ forte, $r>0,5$ moderada, $r<0,5$ fraca, $r=0$ inexistente. $p<0,05$.

Fonte: Autores. 
O objetivo deste trabalho foi avaliar a qualidade de vida de voluntários saudáveis através do instrumento SF-36 por contato telefônico durante a pandemia do COVID-19. O trabalho justifica-se diante do cenário de insegurança e medo vivenciados por toda a população. No presente estudo conduzido durante a pandemia, pôde-se observar que não houve diferença significativa entre homens e mulheres nos domínios do questionário. Este resultado vai de encontro com os achados de Guiraldo e colaboradores (2020), onde compararam os resultados obtidos do questionário SF-36 antes e após a pandemia por COVID-19. Resultado semelhante ao encontrado por Zhang e Ma (2020) onde relataram que a pandemia por COVID-19 foi associada apenas a um leve impacto na qualidade de vida de uma amostra de chineses. Segundo os autores, como a pandemia está em andamento serão necessários novos estudos que confirmem o achado.

Os resultados permitem concluir que, em média, os participantes desta pesquisa, apresentaram escores acima de $50 \mathrm{em}$ todos os domínios que compõe o questionário de qualidade de vida SF-36, demonstrando que os mesmos não estão abaixo da média de qualidade de vida esperada, sendo o desempenho dos participantes do gênero masculino ligeiramente melhor do que as participantes do gênero feminino nos domínios capacidade funcional, dor, aspectos sociais e aspectos emocionais, porém sem diferença significativa entre eles.

\section{Considerações Finais}

Diante do exposto, não houve diferença significativa entre os domínios do questionário SF-36 entre homens e mulheres. Houve diferença significativa entre alguns domínios quando correlacionados, porém com correlação de Pearson linear positiva fraca.

São necessários mais estudos para obter um resultado expressivo. Sugerimos estudos futuros de comparações entre resultados do questionário SF-36 após um período de pandemia por COVID-19 para avaliar antes e após, podendo assim saber se a pandemia alterou a qualidade de vida de uma determinada população.

\section{Agradecimentos}

Nossos sinceros agradecimentos ao Programa de Pós Graduação em Biociências Aplicadas à Saúde da Universidade Federal de Alfenas (UNIFAL), ao Professor Doutor Leonardo César Carvalho (UNIFAL) pelos ensinamentos e ao Centro Superior de Ensino e Pesquisa de Machado/MG (CESEP).

\section{Referências}

Adorno, M. L. G. R. \& Brasil-Neto, J. P. (2013). Avaliação da qualidade de vida com o instrumento SF-36 em lombalgia crônica. Acta Ortop Bras, 21(4): 202-207.

Bashshur, R. (2020). Telemedicine and the COVID-19 pandemic, lessons for the future. Telemed J E Health, 26, $571-573$.

Casonatto J. \& Doederlein M. (2009). Post-exercise Hypotension: a Systematic Review. Rev Bras Med Esporte, Mar/Apr;15, $151-157$.

Chatterjee S. (2020). Important Steps to Control COVID-19/SARS-CoV-2 Infection.SN Compr Clin Med, 1-2.

Ciconelli, R. M et al. (1999). Tradução para a língua portuguesa e validação do questionário genérico de avaliação de qualidade de vida SF-36 (Brasil SF-36). Rev Bras Reumatol, 39(3), 143-50.

Cottrell, M. et al. (2021). Comparing fly-in fly-out and telehealth models for delivering advanced-practice physiotherapy services in regional Queensland: an audit of outcomes and costs. J. Telemed. Telecare. 27(1), 32-38.

Dalfovo, M. S.; Lana, R. A. \& Silveira, A. Métodos quantitativos e qualitativos: um resgate teórico. Revista Interdisciplinar Científica Aplicada, Blumenau, 2(4), 01- 13 .

Guirado, G. M. P. et al. (2021). Avaliação da qualidade de vida de trabalhadores antes e durante a pandemia de COVID-19 por meio do questionário SF-36 Rev. Gest. Sist. Saúde, São Paulo, 10(1), 84-105.

Hollander J. E. \& Carr, B.G. (2020). Virtually perfect? Telemedicine for covid-19. N Engl JMed, 382, 1679-1681. 
Research, Society and Development, v. 10, n. 9, e17210917596, 2021

(CC BY 4.0) | ISSN 2525-3409 | DOI: http://dx.doi.org/10.33448/rsd-v10i9.17596

Mcintosh, K. (2020). Novel Coronavirus (2019-nCov) from https://www.uptodate.com/contents/coronavirus-disease-2019-covid-19

Ministério da Saúde. (2020). Secretaria de Atenção Especializada à Saúde (SAES). Departamento de Atenção Hospitalar, Domiciliar e de Urgência (DAHU). Protocolo de manejo clínico para o novo coronavírus (2019-nCoV). Brasília, 2020. From https://portalarquivos2.saude.gov.br/images/pdf/2020/fevereiro/11/protocolo-manejo-coronavirus

Moretti, S.A.; Guedes-Neta, M.L. \& Batista, E. C. (2020). Nossas Vidas em Meio à Pandemia da COVID - 19: Incertezas e Medos Sociais. Rev Enfermagem e Saúde Coletiva, 4(2), 32-41.

Nelson, M. et al. (2019). Cost-effectiveness of telerehabilitation versus traditional care after total hip replacement: a trial-based economic evaluation. $J$. Telemed. Telecare. https://doi.org/10.1177/1357633X19869796, 1357633X19869796.

Oliveira, V. et al. (2020). COVID-19 e a Transformação Digital dos Cuidados de Saúde: “A Pastilha de Mentos na Coca-Cola Diet”. Gazeta Médica, 7(2).

Pastora-Bernal, J.M., Martín-Valero, R. \& Barón-López, F.J. (2017). Cost analysis of telerehabilitation after arthroscopic subacromial decompression. J. Telemed. Telecare 24 (8), 553-559.

Poulter, N. R.; Prabhakaran D \& Caulfield, M. (2015). Hypertension. Lancet 386, 801-812.

Rockwell, K. L. \& Gilroy, A. S. (2020). Incorporating telemedicine as part of COVID-19 outbreak response systems. Am J Manag Care, $26,147-148$.

Tousignant, M. et al. (2015). Cost analysis of in-home telerehabilitation for post-knee arthroplasty. J. Med. Internet Res. 17 (3) https://doi.org/10.2196/jmir.3844 e83-e83.

Vetta, F.; Vetta, G. \& Marinaccio, L. (2020). Coronavirus disease 2019 (COVID-19) and cardiovascular disease: a vicious circle. J Cardiol Cardiovasc Res, 1, $1-12$.

World Health Organization. (2020). Rolling updates on coronavirus disease (COVID-19) 2020. From https://www.who.int/emergencies/diseases/novelcoronavirus-2019?gclid=EAIaIQobChMIr9Ks562J6gIVTwmRCh1RbwgjEAAYASAAEgK_V_D_BwE

Zhang, Y., \& Ma, Z. F. (2020). Impact of the COVID-19 Pandemic on Mental Health and Quality of Life among Local Residents in Liaoning Province, China: A Cross-Sectional Study. International Journal of Environmental Research and Public Health, 17(7), 2381. 